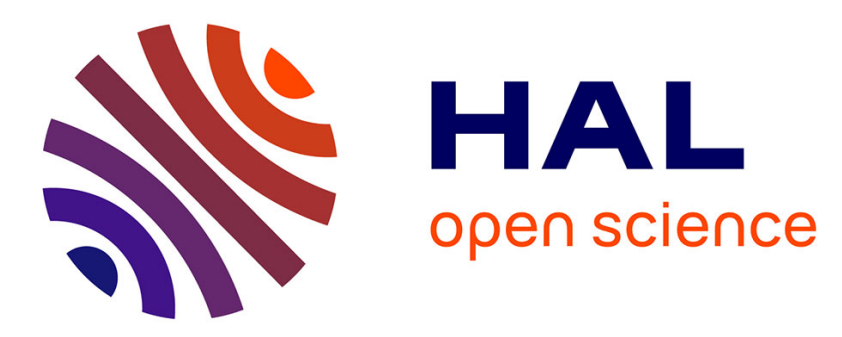

\title{
Caractérisation Raman des contraintes et des défauts d'interface dans GaAs/Si
}

Adnen Mlayah, Robert Carles, Georges Landa, C. Fontaine, A. Freundlich

\section{To cite this version:}

Adnen Mlayah, Robert Carles, Georges Landa, C. Fontaine, A. Freundlich. Caractérisation Raman des contraintes et des défauts d'interface dans GaAs/Si. Revue de Physique Appliquée, 1990, 25 (9), pp.951-956. 10.1051/rphysap:01990002509095100 . jpa-00246262

\section{HAL Id: jpa-00246262 https://hal.science/jpa-00246262}

Submitted on 1 Jan 1990

HAL is a multi-disciplinary open access archive for the deposit and dissemination of scientific research documents, whether they are published or not. The documents may come from teaching and research institutions in France or abroad, or from public or private research centers.
L'archive ouverte pluridisciplinaire HAL, est destinée au dépôt et à la diffusion de documents scientifiques de niveau recherche, publiés ou non, émanant des établissements d'enseignement et de recherche français ou étrangers, des laboratoires publics ou privés. 


\title{
Caractérisation Raman des contraintes et des défauts d'interface dans GaAs/Si
}

\author{
A. Mlayah $\left({ }^{1}\right)$, R. Carles $\left({ }^{1}\right)$, G. Landa $\left({ }^{1}\right)$, C. Fontaine $\left({ }^{2}\right)$ et A. Freundlich $\left({ }^{3}\right)$ \\ ( $\left.{ }^{1}\right)$ Laboratoire de Physique des Solides, U.R.A. 74 du C.N.R.S., Université Paul Sabatier, 118 route de \\ Narbonne, 31062 Toulouse Cedex, France \\ $\left(^{2}\right)$ Laboratoire d'Automatique et d'Analyse des Systèmes, U.P.R. 8001 du C.N.R.S., 7 avenue du Colonel \\ Roche, 31077 Toulouse Cedex, France \\ ( $\left.{ }^{3}\right)$ Laboratoire de Physique du Solide et d'Energie Solaire, U.P.R. 10 du C.N.R.S., Sophia-Antipolis, rue \\ B. Grégory, 06560 Valbonne, France
}

(Reçu le 21 décembre 1989, révisé le 19 février 1990, accepté le 26 février 1990)

\begin{abstract}
Résumé. - La spectroscopie Raman a été utilisée en condition de résonance dans le cas de plusieurs hétérostructures $\mathrm{GaAs} / \mathrm{Si}(100)$, pour tester la qualité cristalline et pour déterminer le signe, la valeur, la nature et l'origine des contraintes dans les couches épitaxiées. La comparaison avec le cas d'un échantillon de $\mathrm{GaAs} / \mathrm{CaF}_{2}$, révèle que le signe et la valeur de la déformation sont essentiellement liés à la différence des coefficients de dilatation thermique de la couche et du substrat. A partir d'une couche de GaAs biseautée, nous avons étudié le profil de la densité de dislocations au voisinage de l'interface, grâce à une analyse des formes de raies Raman des modes longitudinaux et transverses optiques. L'épaisseur de la couche disloquée ainsi déterminée est voisine de $7 \mathrm{~nm}$, cette valeur est en bon accord avec celle, obtenue en suivant l'activation du mode $T O$ interdit, de la couche de nucléation déposée à basse température lors du premier stade du processus de croissance à deux étapes.
\end{abstract}

\begin{abstract}
Raman spectroscopy has been performed under resonant conditions on several $\mathrm{GaAs} / \mathrm{Si}(100)$ heterostructures to probe the crystalline quality, the sign, the value, the nature and the origine of the strain present in the $\mathrm{GaAs}$ layer. The comparison with the case of a $\mathrm{GaAs} / \mathrm{CaF}_{2}$ sample, clearly reveals that the sign and the value of the strain are mainly related to the difference between the thermal expansion coefficients of the layer and the substrate. From a bevel edged GaAs layer, we have studied the misfit dislocations density profile in the vicinity of the interface by means of Raman lineshapes analysis of both longitudinal and transverse optical modes. The thickness of the dislocated layer is found to be about $7 \mathrm{~nm}$, a value which is in agreement with the one determined from the forbidden TO mode activation, of the buffer layer deposited at low temperature during the two step growth process.
\end{abstract}

\section{Introduction : principe de la caractérisation Raman.}

La spectrométrie Raman est une technique qui s'appuie sur un processus optique à deux photons. L'analyse des corrélations entre les photons incidents (énergie $E_{\mathrm{i}}$, vecteur d'onde $\mathbf{k}_{\mathrm{i}}$, vecteur de polarisation $\left.\mathbf{e}_{\mathrm{i}}\right)$ et les photons diffusés $\left(E_{\mathrm{d}}, \mathbf{k}_{\mathrm{d}}, \mathbf{e}_{\mathrm{d}}\right)$ permet de caractériser les excitations élémentaires créées (processus Stokes) lors de l'interaction lumière-matériau et à travers elles le matériau lui-même.

Ces corrélations sont traduites par des lois de conservation et des règles d'activité. Expérimentalement, une raie Raman livre trois informations: sa fréquence, sa largeur de raie (ou plus précisément sa forme) et sa polarisation.
Sa fréquence $\omega$, déduite de la loi de conservation :

$$
E_{\mathrm{i}}-E_{\mathrm{d}}=\hbar \omega,
$$

est une caractéristique de la dynamique de réseau du matériau. Toute modification $\Delta \omega$ de cette fréquence permet de déduire le signe et l'amplitude d'une contrainte éventuellement présente [1]. La mesure comparée des décalages sur différents modes de vibration renseigne sur l'anisotropie de celle-ci.

La largeur de raie correspondante $\Gamma$ permet d'évaluer la longueur de corrélation $L$ du mode considéré $[2,3]$. En effet, le vecteur de diffusion :

$$
\mathbf{k}=\mathbf{k}_{\mathrm{i}}-\mathbf{k}_{\mathrm{d}},
$$


étant quasi-nul, seuls les modes de grande longueur d'onde sont observables. La présence de défauts (dislocations, impuretés) rompt la symétrie de translation: le mode observé n'est plus un phonon (onde plane) à $\mathbf{q}=\mathbf{0}$ de la zone de Brillouin d'un monocristal, mais un paquet d'ondes dont les vecteurs d'onde q sont représentatifs d'un volume de cette zone, dont l'extension croît avec la densité des défauts. En première approximation, cette extension dans l'espace réciproque est de l'ordre de :

$$
\Delta k=2 \pi / L,
$$

$L$ étant directement lié à la distance moyenne entre défauts. Grâce à la connaissance des courbes de dispersion $\omega(\mathbf{q}), L$ est calculable à partir de l'élargissement intrinsèque, de l'asymétrie de la raie Raman correspondante [2].

Enfin, la polarisation de cette raie, liée à la symétrie du mode de vibration et au mécanisme d'interaction, peut être déduite de l'étude des règles de sélection. Toute modification de l'orientation, due par exemple au maclage [4], où toute perte de symétrie se traduit alors par l'activation de modes qualifiés d'interdits par référence au cas d'un cristal parfait. Ainsi, en rétrodiffusion sur une face (100) d'un cristal de GaAs, seul le mode longitudinal optique $(L O)$ est observable; a contrario, le mode transverse optique $(T O)$ est seul observable pour une face (110).

\section{Conditions expérimentales.}

Nous avons mis à profit ces possibilités d'analyse spectroscopique pour étudier la présence, la nature, l'origine et la relaxation des contraintes d'interface dans les hétérostructures $\mathrm{GaAs} / \mathrm{Si}(100)$. Ainsi est présenté et interprété un ensemble de résultats expérimentaux relatifs à des échantillons dont les caractéristiques sont diverses quant à leur épaisseur et à leur technique d'élaboration : épitaxie par jets moléculaires (EJM) ou en phase vapeur (EPVOM). Le but de cet article est de tester la sensibilité de la technique et de dégager une série d'effets intrinsèques liés à la différence des paramètres physiques de $\mathrm{GaAs}$ et de $\mathrm{Si}$, indépendamment de la technique et des conditions de croissance.

L'évolution des coefficients d'absorption $\alpha_{1}$ et $\alpha_{2}$ de $\mathrm{GaAs}$ et de $\mathrm{Si}$, en fonction de l'énergie [5] est reportée sur la figure 1. Dans un processus Raman une géométrie de rétrodiffusion est imposée par la forte absorption de ces matériaux et le volume exploré est ainsi limité à une profondeur Raman:

$$
d=\left(\alpha_{\mathrm{i}}+\alpha_{\mathrm{d}}\right)^{-1} \simeq 1 /(2 \alpha) .
$$

Les valeurs de $d$ reportées sur la figure 1 montrent qu'une topographie en épaisseur, bien que différentielle, est a priori possible sur ce type de matériau.

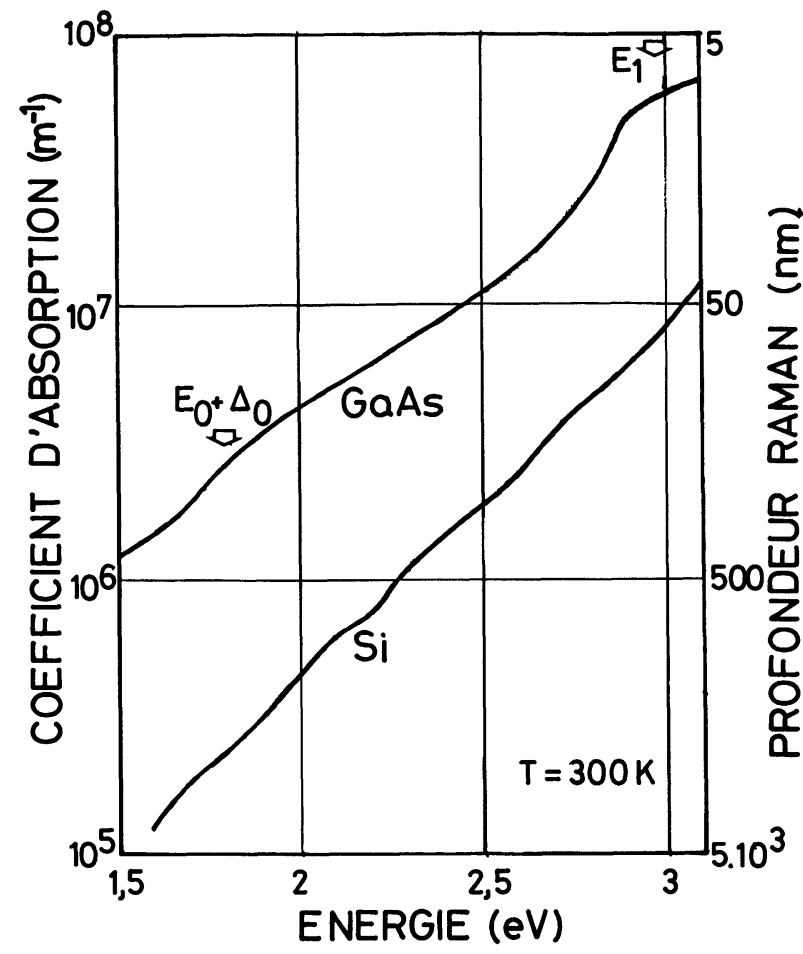

Fig. 1. - Variation des coefficients d'absorption en fonction de l'énergie pour $\mathrm{GaAs}$ et $\mathrm{Si}$. Les profondeurs Raman sont indiquées sur la figure.

[Absorption coefficients for GaAs and $\mathrm{Si}$ versus energy. Raman depth are also indicated.]

L'originalité des mesures présentées tient en fait à l'utilisation d'une énergie excitatrice de $3 \mathrm{eV}$ (raie $413,1 \mathrm{~nm}$ d'un laser $\mathrm{Kr}^{+}$) qui présente dans le cas de GaAs, un double avantage : d'une part de limiter la profondeur $d$ à $8,5 \mathrm{~nm}$ et d'autre part de bénéficier de conditions de résonance au voisinage de la transition optique $E_{1}(2,985 \mathrm{eV}$ à $300 \mathrm{~K})$. L'exaltation de la section efficace de diffusion qui en résulte permet, malgré un faible volume de diffusion, d'éviter un échauffement notable de l'échantillon tout en maintenant une bonne résolution $\left(2,15 \mathrm{~cm}^{-1}\right)$.

\section{Nature et évaluation des contraintes.}

3.1 RÈGleS DE SÉLECTION. - Sur la figure 2, sont reportés les spectres Raman relatifs à la bande optique de GaAs pour un échantillon épais $(e=4 \mu \mathrm{m})$ de $\mathrm{GaAs} / \mathrm{Si}(100)$ et obtenus dans des conditions non résonnantes $\left(E_{\mathrm{i}}=2,54 \mathrm{eV}\right)$, en vraie rétrodiffusion, pour quatre configurations différentes des vecteurs de polarisation. Le phonon $L O$ de symétrie $\Gamma_{15}$ n'apparaît comme prévu que dans les deux configurations où cette composante est autorisée [4]. D'autre part, aucun signal correspondant au mode $T O$ de nombre d'onde voisin de $269 \mathrm{~cm}^{-1}$ n'est enregistré. Cette stricte obéissance aux règles 


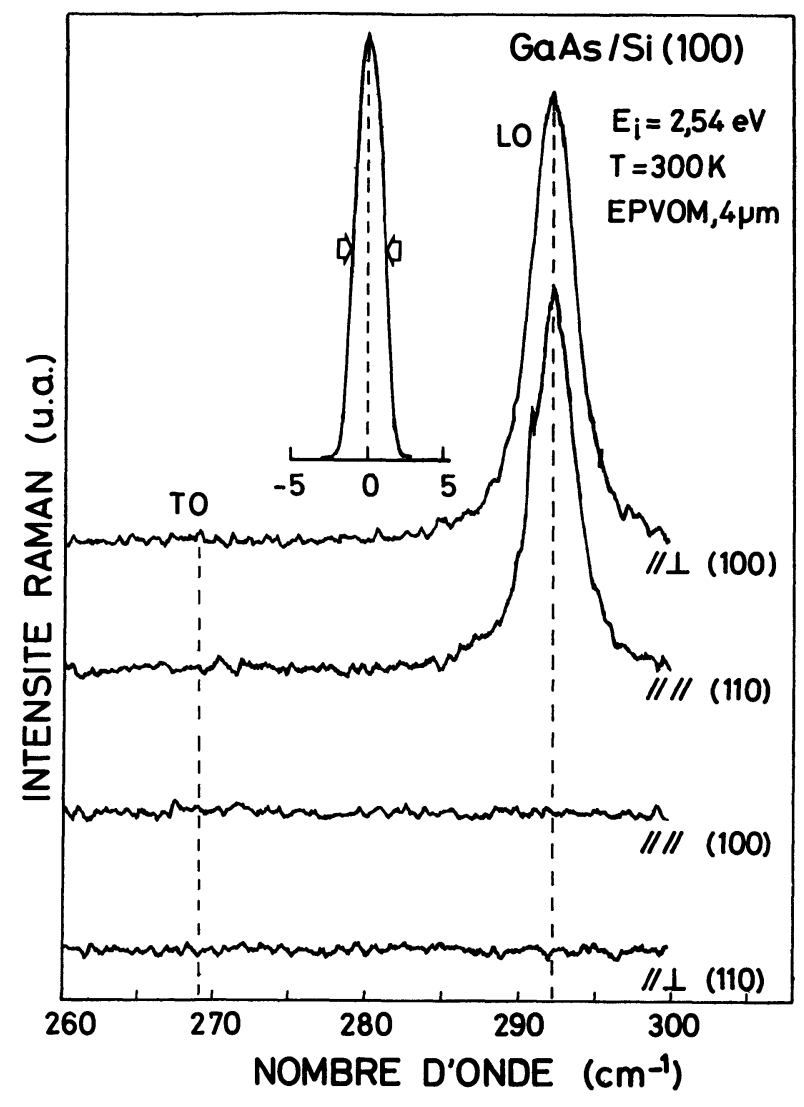

Fig. 2. - Règles de sélection pour différentes configurations des vecteurs polarisation. Le pic de diffusion Rayleigh montre la résolution de l'appareil.

[Selection rules for different polarisation vectors configurations. The resolution of the apparatus is showned by the Rayleigh peak.]

de sélection prouve la bonne qualité cristalline de la couche de GaAs qui, dans la limite de la sensibilité Raman, est comparable à celle d'une homoépitaxie.

3.2 VAleurs DES CONTRAinTES. - Les effets de désordre s'avérant négligeables pour la diffusion Raman par des couches suffisamment épaisses (supérieures à $1 \mu \mathrm{m}$ ), la manifestation des contraintes résiduelles peut être directement déduite des décalages de raies. La figure 3 montre que ceux-ci étant très faibles, ils sont évalués par comparaison avec le spectre relatif à une homoépitaxie enregistré dans les mêmes conditions expérimentales $(T=77 \mathrm{~K}$, $E_{\mathrm{i}}=3 \mathrm{eV}$ ). Les spectres ont été obtenus dans le cas d'une incidence oblique, quasi-brewstérienne, qui permet ainsi d'activer le mode $T O$, le vecteur de diffusion ayant une composante selon [011]. D'autre part, l'excitation en condition de résonance permet l'observation de la diffusion au second ordre sous forme d'harmoniques de modes $L O$.

Les décalages vers les basses fréquences des trois raies Raman révèlent la présence d'une contrainte extensive dans la couche hétéroépitaxiée. L'ampli-

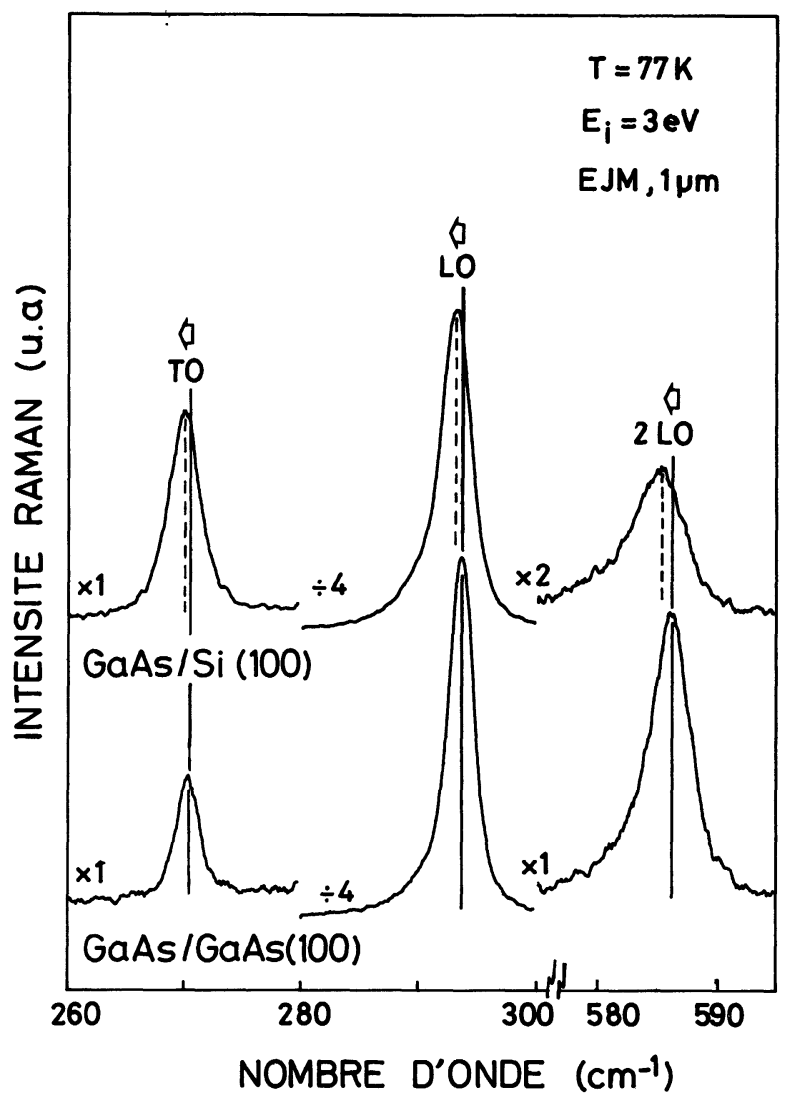

Fig. 3. - Comparaison de la diffusion par le premier et second ordre optique entre l'homoépitaxie (figure du bas) et l'hétéroépitaxie (figure du haut).

[First and second order optical scattering comparison between homoepitaxy (lower part) and heteroepitaxy (upper part).]

tude de cette contrainte peut être évaluée quantitativement selon [1] :

$$
\begin{aligned}
& \Delta \omega\left(L O_{\mathrm{S}}\right)=-0,49 \times 10^{3} \varepsilon_{\|} \\
& \Delta \omega\left(T O_{\mathrm{D}}\right)=-0,34 \times 10^{3} \varepsilon_{\|} .
\end{aligned}
$$

La levée de la dégénérescence cubique due à la contrainte permet d'associer à un mode optique donné, un mode dit doublet (D) et un mode singulet (S). L'analyse des règles de sélection [1] prédit dans le cas d'une déformation uniaxiale selon la direction de croissance que le mode $L O$ est de type singulet alors que le mode $T O$ est de type doublet. Cette hypothèse est confirmée expérimentalement par l'observation d'un décalage plus faible, selon les formules ci-dessus, pour le mode TO. A partir des valeurs expérimentales :

$$
\Delta \omega\left(L O_{\mathrm{S}}\right)=-0,6 \pm 0,1 \mathrm{~cm}^{-1}
$$

et

$$
\Delta \omega\left(T O_{\mathrm{D}}\right)=-0,4 \pm 0,1 \mathrm{~cm}^{-1},
$$


on déduit [1] les valeurs de la déformation dans le plan, au voisinage de la surface, et la contrainte $X$ associée, soit :

$$
\varepsilon_{\|}=(1,2 \pm 0,2) \cdot 10^{-3}
$$

et

$$
X=(0,15 \pm 0,03) \mathrm{GPa} .
$$

L'analyse du spectre du second ordre, originale dans une expérience de caractérisation, vient étayer les résultats précédents. En effet, le mode $2 L O$ enregistré dans des conditions de résonance, correspond en fait à des harmoniques de modes $1 L O(\Gamma)$, le mécanisme d'interaction étant du type Fröhlich intrabande [6]. Une estimation du décalage en fréquence peut être menée en tenant compte de la présence combinée des deux types de modes (D et $S$ ) et de leurs dégénérescences respectives. La différence entre $\Delta \omega\left(L O_{\mathrm{S}}\right)$ et $\Delta \omega\left(L O_{\mathrm{D}}\right)$ étant très faible devant la largeur de raie liée aux effets thermiques et à la présence de défauts, seul le décalage du barycentre de la raie $2 L O$ est mesurable. Sa valeur est calculée selon :

$$
\Delta \omega(2 L O) \simeq 2\left[\Delta \omega\left(L O_{\mathrm{S}}\right)+2 \Delta \omega\left(L O_{\mathrm{D}}\right)\right] / 3 .
$$

Le décalage mesuré de $(1,0 \pm 0,2) \mathrm{cm}^{-1}$ conduit à une valeur de $\varepsilon_{\|} \simeq(1,4 \pm 0,3) \cdot 10^{-3}$ en bon accord avec l'estimation précédente.

L'amplitude de la contrainte dépend des conditions de croissance et de l'épaisseur des couches, mais il convient d'en préciser l'origine.

3.3 ORIGINE DES CONTRAINTES. - La nature extensive de la contrainte ne peut provenir dans son ensemble du désaccord de maille de $4 \%$ entre GaAs et $\mathrm{Si}$, celui-ci conduisant à une compression dans les plans parallèles à l'interface. La spectrométrie Raman parmi d'autres techniques (photoluminescence, diffraction $\mathrm{X}$ ) a permis de montrer que la contrainte était très dépendante de la température, et que cette dépendance était imputable à la différence des coefficients d'expansion thermique entre les deux matériaux. Cette contribution dominante à température ambiante [1] est mise en évidence de manière originale sur la figure 4 où sont comparés les spectres de couches de GaAs déposés sur différents substrats. En particulier, bien que le désaccord de maille dans les deux systèmes $\mathrm{GaAs} / \mathrm{Si}$ et $\mathrm{GaAs} / \mathrm{CaF}_{2}$ soient comparables en signe et amplitude ( $4 \%$ et $3,8 \%$, respectivement), les décalages des raies et donc les déformations sont de signe et d'amplitude différents $\left(1,5 \times 10^{-3}\right.$ et $-5,6 \times 10^{-3}$, respectivement). Ces résultats sont en revanche conformes aux différences de leurs coefficients d'expansion thermique $\left(3,4 \times 10^{-6}\right.$ et $-13 \times 10^{-6} \mathrm{~K}^{-1}$, respectivement)

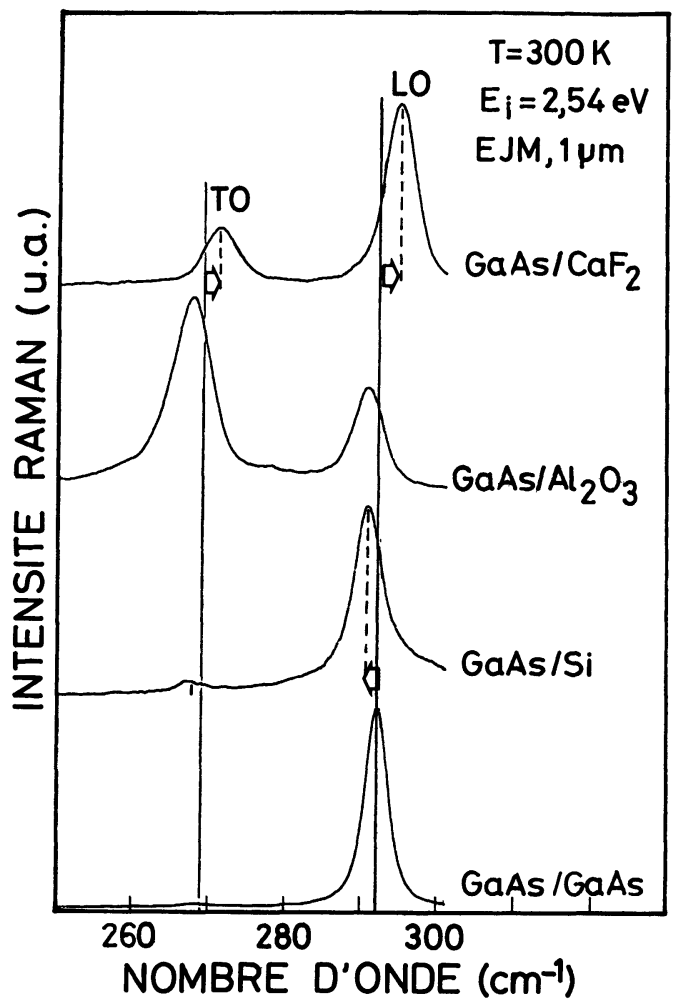

Fig. 4. - Influence de la différence des coefficients d'expansion thermique sur la diffusion au premier ordre pour diverses hétéroépitaxies.

[Influence of the difference of thermal expansion coefficients on the first order scattering for various heteroepitaxies.]

\section{Relaxation des contraintes et défauts associés.}

4.1 ACTIVATION RAMAN PAR LES DÉFAUTS CRISTALLINS. - La présence d'une bande DATO (TO Activé par le Désordre) en rétrodiffusion, l'élargissement des raies et leur asymétrie, l'atténuation des structures du second ordre par rapport à celle du premier ordre sont autant de manifestations de la rupture de la symétrie de translation dans le cristal. Dans le cas de $\mathrm{GaAs} / \mathrm{Si}$, ces paramètres ont été largement utilisés pour un suivi de l'amélioration des conditions de croissance [7-9]. Un nouvel exemple est donné pour une série d'échantillons qui ne diffèrent que par l'épaisseur de la couche de nucléation déposée dans la première étape de croissance [7]. La corrélation entre l'activation Raman du mode $D A T O$ par rapport à celle du mode $L O$ et le taux de dislocations révélées chimiquement [7] est très nette, comme en témoignent les résultats reportés dans le tableau I. Ainsi, la sonde Raman permet de préciser que l'épaisseur optimale de la couche initiale permettant dans ce processus de croissance (EPVOM) une minimisation du taux de dislocations se situe aux environs de $8 \mathrm{~nm}$. 
Tableau I. - Taux de dislocations, rapports des intensités TO/LO et décalages du mode LO en fonction de l'épaisseur de la couche de nucléation.

[Dislocation densities, TO/LO intensities ratio and $L O$ shifts versus nucleation layer thickness.]

\begin{tabular}{|c|c|c|c|}
\hline$e(\mathrm{~nm})$ & $d\left(\mathrm{~cm}^{-2}\right)$ & $I_{T O} / I_{L O}(\%)$ & $\Delta \omega_{L O}\left(\mathrm{~cm}^{-1}\right)$ \\
\hline 4 & $5,9 \times 10^{7}$ & 14 & $0,7 \pm 0,1$ \\
\hline 8 & $3,4 \times 10^{7}$ & 5 & $0,8 \pm 0,1$ \\
\hline 20 & $9,0 \times 10^{7}$ & 34 & $0,6 \pm 0,1$ \\
\hline
\end{tabular}

\subsection{EVOLUTION DU NOMBRE DE DÉFAUTS AVEC} L'ÉPAISSEUR. - Selon une procédure déjà utilisée [10] pour ce type d'hétérostructure mais dont nous avons amélioré la résolution spatiale et spectrale, une série de mesures Raman a été réalisée à partir d'un échantillon ( $1 \mu \mathrm{m}, \mathrm{EJM}$, surface vicinale désorientée de $2^{\circ}$ par rapport à $\langle 100\rangle$ vers $\langle 110\rangle$ ) biseauté $\left(\beta=10^{-3}\right)$ par attaque chimique (cf. Fig. 5). Au voisinage de l'interface, les signaux du substrat de $\mathrm{Si}$ et de la couche de GaAs sont simultanément présents ; l'évolution de leurs intensités relatives est reportée sur la figure 5. Moyennant une modélisation [11] qui prend en compte les propriétés physiques des matériaux et toutes les caractéristiques expérimentales, on peut rendre compte de ces évolutions, comme en témoignent les courbes théoriques reportées sur cette même figure. Ce premier test permettant de contrôler les caractéristiques de l'échantillon (position de l'interface, épaisseur, absorption, section efficace de diffusion), l'évolution de la densité de défauts en fonction de la distance $z$ à l'interface peut alors être abordée. Celle-ci se déduit de l'évolution des formes de raies. A titre d'exemple, est reportée sur la figure 6 celle de la largeur intrinsèque $\Gamma$ (après déconvolution de la fonction d'appareil) des raies $L O$ et $T O$ : une même décroissance de type exponentiel rend compte pour les deux modes du recouvrement rapide de la symétrie de translation, liée à la diminution de la densité de défauts, selon:

$$
\Delta \Gamma(z)=\Delta \Gamma(0) \exp (-z / \delta),
$$

avec

$$
\Delta \Gamma(z)=\Gamma(z)-\Gamma(\infty) .
$$

La valeur caractéristique $\delta$ déduite de ce type d'ajustement est voisine de $7 \mathrm{~nm}$; elle donne un bon ordre de grandeur de la distance minimale nécessaire pour absorber la majorité des dislocations créées à l'interface par le désaccord paramétrique. On peut remarquer que cette valeur est très proche de

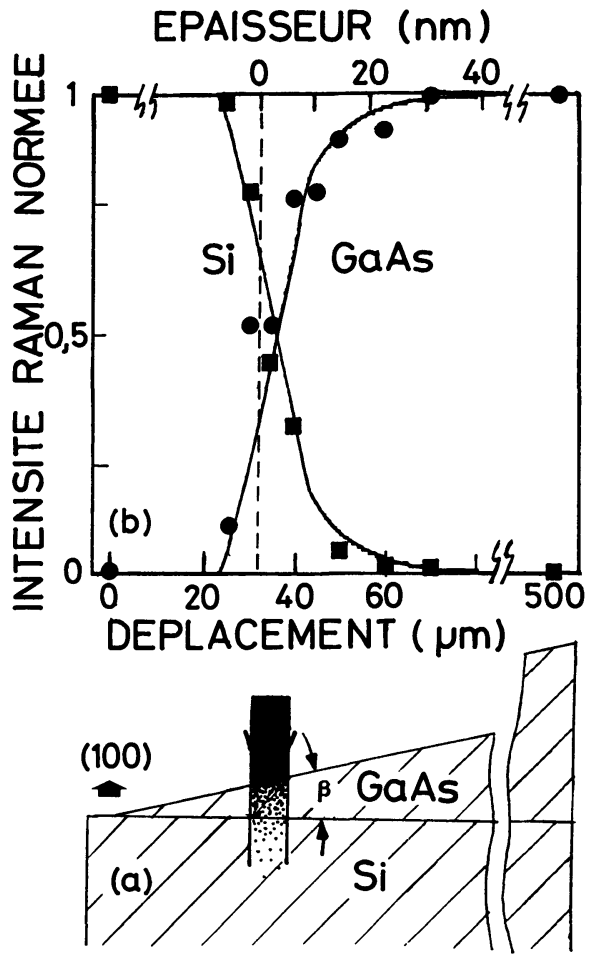

Fig. 5. - a) Coupe longitudinale de l'échantillon biseauté montrant la géométrie de diffusion. b) Variation des intensités Raman normées associées au Si et au GaAs en fonction du déplacement sur le biseau. L'épaisseur de la couche de GaAs est aussi indiquée.

(a) Longitudinal cross-section of the beveled sample showing the scattering geometry. b) Variation of the normalized Raman intensities for the $\mathrm{Si}$ and $\mathrm{GaAs}$ peaks versus the position on the bevel. The thickness of the GaAs layer is also indicated.]

l'épaisseur optimale $(8 \mathrm{~nm})$ de la couche de nucléation nécessaire pour obtenir une bonne qualité cristalline loin de l'interface. Enfin, l'analyse des formes de raie par un modèle de corrélation spatiale que nous avons adapté au cas présent [11] permet de déterminer les distances dans le plan de l'interface sur lesquelles les mouvements des atomes restent corrélés. A partir de résultats déjà publiés concernant des problèmes d'implantation [2], une valeur de $\Gamma_{L O}(0)=3,7 \mathrm{~cm}^{-1}$ permet d'estimer cette distance à environ $10 \mathrm{~nm}$, valeur en bon accord avec la distance inter-dislocations nécessaire pour rattraper le désaccord de maille.

\section{Conclusion.}

L'ensemble des résultats de spectrométrie Raman obtenus sur de nombreux échantillons de $\mathrm{GaAs} / \mathrm{Si}(100)$ confirme la bonne adaptation de cette technique à une caractérisation cristalline de ce type d'hétérostructures. Nous avons montré l'intérêt de travailler en condition de résonance (faible échauffe- 


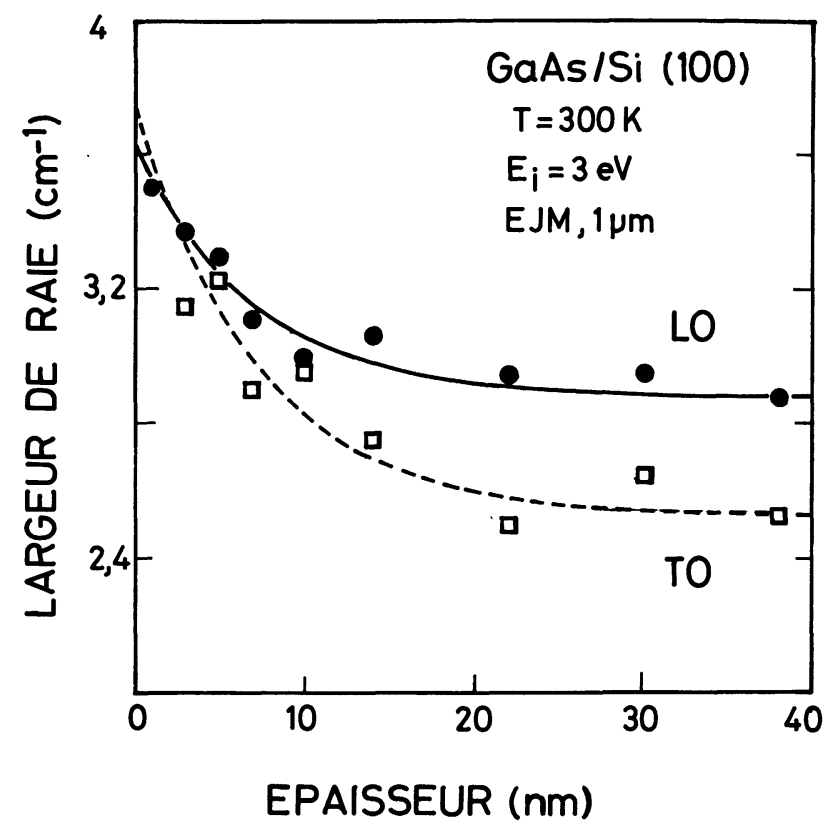

Fig. 6. - Variation de la largeur des modes transverses optiques $(\square)$ et longitudinaux optiques $(\bullet)$ en fonction de la distance à l'interface.

[Variation of transverse ( $\square$ ) and longitudinal ( $\bullet$ ) optical mode widths versus the distance from the interface.]

ment, forte efficacité de diffusion, Raman du second ordre), avec une profondeur d'analyse réduite (topographie en épaisseur), en lumière polarisée puis analysée (activation de structures interdites par les défauts) et en variant l'angle d'incidence (analyse comparée sur plusieurs modes). Il convient de noter que la sonde Raman n'est sensible qu'à des taux de défauts relativement élevés. Au-delà d'une distance inter-défauts supérieure à quelques dizaines de $\mathrm{nm}$, l'effet sur les largeurs de raie devient négligeable. Son efficacité est limitée à l'étude de matériaux fortement perturbés, comme c'est le cas au voisinage immédiat d'interfaces à fort désaccord paramétrique.

Ainsi un certain nombre de caractéristiques ont pu être confirmées voire démontrées: la contrainte extensive présente un caractère biaxial dans le plan de croissance, dont la valeur est essentiellement liée, à température ambiante, à la différence entre les coefficients d'expansion thermique. Le désaccord paramétrique se traduit par un taux très élevé de dislocations d'interface qui décroît très rapidement avec la distance, comme en témoigne la décroissance exponentielle des largeurs de raie avec un paramètre caractéristique inférieur à $10 \mathrm{~nm}$. Bien que les couches conservent une contrainte de l'ordre de $0,15 \mathrm{GPa}$, une bonne qualité cristalline (comparable du point de vue de la spectrométrie Raman à celle d'une homoépitaxie) est obtenue avec des épaisseurs de couches de l'ordre de $1 \mu \mathrm{m}$, c'est-à-dire compatibles avec de nombreuses applications.

\section{Remerciements.}

Les auteurs remercient A. Muñoz-Yagüe et A. Rocher pour les nombreuses discussions sur ce travail.

\section{Bibliographie}

[1] Landa G., Carles R., Fontaine C., Bedel E. and Muñoz-Yagüe A., J. Appl. Phys. 66 (1989) 196.

[2] Tiong K. K., Amirtharaj P. M., Pollak F. H. and Aspnes D. E., Appl. Phys. Lett. 44 (1984) 122.

[3] Maury F., Carles R., Landa G. and RenuCCI J. B., Thin Solid Films 155 (1987) 331.

[4] Landa G., Carles R., Renucci J. B., Fontaine C., Bedel E. and Muñoz-Yagüe A., J. Appl. Phys. 60 (1986) 1025.

[5] Aspnes D. E. and Studna A. A., Phys. Rev. B 27 (1983) 985.

[6] Trommer R. and Cardona M., Phys. Rev. B 17 (1978) 1865 .
[7] Freundlich A., Grenet J. C., Neu G., Leycuras A., Vérié C., Gibart P., Landa G. and CARles R., J. Cryst. Growth 93 (1988) 487.

[8] Freundlich A., Neu G., Leycuras A., Carles R. and Vérié C., Proc. Mat. Research. Soc. Spring Meeting (Reno, U.S.A., 1988) 9.

[9] Fontaine C., Benarfa H., Bedel E., MuñozYagǘ A., LANda G. and Carles R., J. Appl. Phys. 60 (1986) 208.

[10] Huang Y., Yu P., Charasse M. N., Lo Y. and Wang S., Appl. Phys. Lett. 51 (1987) 192.

[11] Mlayah A., Carles R., landa G., Bedel E., FONTAINE C. and MUÑOZ-YAGÜE (à paraître). 